Research Article

\title{
Perturbation Analysis of the Nonlinear Matrix Equation $X-\sum_{i=1}^{m} A_{i}^{*} X^{p_{i}} A_{i}=\mathrm{Q}$
}

\author{
Jing Li \\ School of Mathematics and Statistics, Shandong University, Weihai 264209, China \\ Correspondence should be addressed to Jing Li; xlijing@sdu.edu.cn
}

Received 15 March 2013; Accepted 7 May 2013

Academic Editor: Vejdi I. Hasanov

Copyright (c) 2013 Jing Li. This is an open access article distributed under the Creative Commons Attribution License, which permits unrestricted use, distribution, and reproduction in any medium, provided the original work is properly cited.

Consider the nonlinear matrix equation $X-\sum_{i=1}^{m} A_{i}^{*} X^{p_{i}} A_{i}=Q$ with $0<p_{i}<1$. Two perturbation bounds and the backward error of an approximate solution to the equation are derived. Explicit expressions of the condition number for the equation are obtained. The theoretical results are illustrated by numerical examples.

\section{Introduction}

In this paper we consider the Hermitian positive definite solution of the nonlinear matrix equation

$$
X-\sum_{i=1}^{m} A_{i}^{*} X^{p_{i}} A_{i}=Q
$$

where $0<p_{i}<1(i=1,2, \ldots, m), A_{1}, A_{2}, \ldots, A_{m}$ are $n \times n$ complex matrices, $m$ is a positive integer, and $Q$ is a positive definite matrix. Here, $A_{i}^{*}$ denotes the conjugate transpose of the matrix $A_{i}$.

When $m>1,(1)$ is recognized as playing an important role in solving a system of linear equations. For example, in many physical calculations, one must solve the system of linear equation

$$
M x=f
$$

where

$$
M=\left(\begin{array}{ccccc}
I & 0 & \cdots & 0 & A_{1} \\
0 & I & \cdots & 0 & A_{2} \\
\vdots & \vdots & \ddots & \vdots & \vdots \\
0 & 0 & \cdots & I & A_{m} \\
A_{1}^{*} & A_{2}^{*} & \cdots & A_{m}^{*} & -Q
\end{array}\right)
$$

arises in a finite difference approximation to an elliptic partial differential equation (for more information, refer to [1]). We can rewrite $M$ as $M=\widetilde{M}+D$, where

$$
\begin{gathered}
\widetilde{M}=\left(\begin{array}{ccccc}
X^{-p_{1}} & 0 & \cdots & 0 & A_{1} \\
0 & X^{-p_{2}} & \cdots & 0 & A_{2} \\
\vdots & \vdots & \ddots & \vdots & \vdots \\
0 & 0 & \cdots & X^{-p_{m}} & A_{m} \\
A_{1}^{*} & A_{2}^{*} & \cdots & A_{m}^{*} & -Q
\end{array}\right), \\
D=\left(\begin{array}{ccccc}
I-X^{-p_{1}} & 0 & \cdots & 0 & 0 \\
0 & I-X^{-p_{2}} & \cdots & 0 & 0 \\
\vdots & \vdots & \ddots & \vdots & \vdots \\
0 & 0 & \cdots & I-X^{-p_{m}} & 0 \\
0 & 0 & \cdots & 0 & 0
\end{array}\right) .
\end{gathered}
$$

$\widetilde{M}$ can be factored as

$$
\widetilde{M}=\left(\begin{array}{ccccc}
-I & 0 & \cdots & 0 & 0 \\
0 & -I & \cdots & 0 & 0 \\
\vdots & \vdots & \ddots & \vdots & \vdots \\
0 & 0 & \cdots & -I & 0 \\
-A_{1}^{*} X^{p_{1}} & -A_{2}^{*} X^{p_{2}} & \cdots & -A_{m}^{*} X^{p_{m}} & -I
\end{array}\right)
$$




$$
\times\left(\begin{array}{ccccc}
-X^{-p_{1}} & 0 & \cdots & 0 & -A_{1} \\
0 & -X^{-p_{2}} & \cdots & 0 & -A_{2} \\
\vdots & \vdots & \ddots & \vdots & \vdots \\
0 & 0 & \cdots & -X^{-p_{m}} & -A_{m} \\
0 & 0 & \cdots & 0 & X
\end{array}\right)
$$

if and only if $X$ is a solution of equation $X-\sum_{i=1}^{m} A_{i}^{*} X^{p_{i}} A_{i}=$ $Q$. When $m=1$, this type of nonlinear matrix equations arises in ladder networks, dynamic programming, control theory, stochastic filtering, statistics, and so forth [2-7].

For the similar equations $X \pm A^{*} X^{-p} A=Q, X^{s} \pm$ $A^{*} X^{-t} A=Q, X+\sum_{i=1}^{m} A_{i}^{*} X^{-1} A_{i}=I$, and $X=Q-A^{*} X^{-1} A+$ $B^{*} X^{-1} B$, there were many contributions in the literature to the theory, numerical solutions, and perturbation analysis [832]. Jia and Gao [33] derived two perturbation estimates for the solution of the equation $X-A^{*} X^{q} A=Q$ with $0<$ $q<1$. In addition, Duan et al. [34] proved that the equation $X-\sum_{i=1}^{m} A_{i}^{*} X^{\delta_{i}} A_{i}=Q \quad\left(0<\left|\delta_{i}\right|<1\right)$ has a unique positive definite solution. They also proposed an iterative method for obtaining the unique positive definite solution. However, to our best knowledge, there has been no perturbation analysis for (1) with $m>1$ in the known literature.

As a continuation of the previous results, the rest of the paper is organized as follows. In Section 2, some preliminary lemmas are given. In Section 3, two perturbation bounds for the unique solution to (1) are derived. Furthermore, in Section 4, we obtain the backward error of an approximate solution to (1). In Section 5, we also discuss the condition number of the unique solution to (1). Finally, several numerical examples are presented in Section 6.

We denote by $\mathscr{C}^{n \times n}$ the set of $n \times n$ complex matrices, by $\mathscr{H}^{n \times n}$ the set of $n \times n$ Hermitian matrices, by $I$ the identity matrix, by $\mathbf{i}$ the imaginary unit, by $\|\cdot\|$ the spectral norm, by $\|\cdot\|_{F}$ the Frobenius norm, and by $\lambda_{\max }(M)$ and $\lambda_{\min }(M)$ the maximal and minimal eigenvalues of $M$, respectively. For $A=\left(a_{1}, \ldots, a_{n}\right)=\left(a_{i j}\right) \in \mathscr{C}^{n \times n}$ and a matrix $B, A \otimes B=$ $\left(a_{i j} B\right)$ is a Kronecker product, and vec $A$ is a vector defined by vec $A=\left(a_{1}^{T}, \ldots, a_{n}^{T}\right)^{T}$. For $X, Y \in \mathscr{H}^{n \times n}$, we write $X \geq$ $Y(X>Y$, resp.) if $X-Y$ is Hermitian positive semidefinite (definite, resp.).

\section{Preliminaries}

Lemma 1 (see [35]). If $A \geq B>0$ and $0 \leq \gamma \leq 1$, then $A^{\gamma} \geq B^{\gamma}$.

Lemma 2 (see [33]). For any Hermitian positive definite matrix $X$ and Hermitian matrix $\Delta X$, one has

(i) $X^{q}=(\sin q \pi / \pi) \int_{0}^{\infty} X^{1 / 2}(\lambda I+X)^{-1} X^{1 / 2} \lambda^{q-1} d \lambda, 0<$ $q<1$

(ii) $X^{q}=(\sin q \pi /(1-q) \pi) \int_{0}^{\infty} X^{1 / 2}(\lambda I+X)^{-1} X(\lambda I+$ $X)^{-1} X^{1 / 2} \lambda^{q-1} d \lambda, 0<q<1$.

In addition, if $X+\Delta X \geq(1 / \nu) X>0$ and $0<q<1$, then (iii) $\left\|X^{-1 / 2} A^{*}\left((X+\Delta X)^{q}-X^{q}\right) A X^{-1 / 2}\right\| \leq q\left(\| X^{-1 / 2}\right.$ $\left.\left.\Delta X X^{-1 / 2} \|\right)+v\left\|X^{-1 / 2} \Delta X X^{-1 / 2}\right\|^{2}\right)\left\|X^{q / 2} A X^{-1 / 2}\right\|^{2}$.

Lemma 3 (see [34]). The matrix equation $X-\sum_{i=1}^{m} A_{i}^{*} X^{\delta_{i}} A_{i}=$ $Q \quad\left(0<\left|\delta_{i}\right|<1\right)$ always has a unique positive definite solution $X$. The matrix sequence $X_{k}$ :

$$
X_{s+m+1}=Q+\sum_{i=1}^{m} A_{i}^{*} X_{s+i}^{\delta_{i}} A_{i}, \quad s=0,1,2, \ldots,
$$

converges to the unique positive definite solution $X$ for arbitrary initial positive definite matrices $X_{1}, X_{2}, \ldots, X_{m}$.

\section{Perturbation Bounds}

Here the perturbed equation

$$
\widetilde{X}-\sum_{i=1}^{m} \widetilde{A}_{i}^{*} \widetilde{X}^{p_{i}} \widetilde{A}_{i}=\widetilde{Q}
$$

is considered, where $0<p_{i}<1$ and $\widetilde{A_{i}}$ and $\widetilde{Q}$ are small perturbations of $A_{i}$ and $Q$ in (1), respectively. We assume that $X$ and $\widetilde{X}$ are the solutions of (1) and (7), respectively. Let $\Delta X=\widetilde{X}-X, \Delta Q=\widetilde{Q}-Q$ and $\Delta A_{i}=\widetilde{A_{i}}-A_{i}$.

By Lemma 3, we know that (1) always has a unique positive definite solution $X$; then in this section two perturbation bounds for the unique positive definite solution of (1) are developed. The relative perturbation bound in Theorem 5 does not depend on any knowledge of the actual solution $X$ of (1). Furthermore, a sharper perturbation bound in Theorem 8 is derived. lemma.

To prove the next theorem, we first verify the following

Lemma 4. If $X$ is a solution of (1), then

$$
X \geq\left(\lambda_{\min }(Q)+\sum_{i=1}^{m} \lambda_{\min }\left(A_{i}^{*} A_{i}\right) \lambda_{\min }^{p_{i}}(Q)\right) I=\beta I .
$$

Proof. By Lemma 3, (1) with $0<p_{i}<1$ always has a unique positive definite solution $X$. Then $X>0$, and it follows that $X^{p_{i}}>0$. Therefore $X \geq Q$. By Lemma 1 and (1), we have $X \geq$ $Q+\sum_{i=1}^{m} A_{i}^{*} Q^{p_{i}} A_{i} \geq\left(\lambda_{\min }(Q)+\sum_{i=1}^{m} \lambda_{\text {min }}\left(A_{i}^{*} A_{i}\right) \lambda_{\text {min }}^{p_{i}}(Q)\right) I=$ $\beta I$.

The next theorem generalizes [33, Theorem 4] with $m=$ $1,\|\Delta Q\|=0$ to arbitrary integer $m \geq 1,\|\Delta Q\|>0$.

Theorem 5. Let $b=\beta+\|\Delta Q\|-\sum_{i=1}^{m} p_{i} \beta^{p_{i}}\left\|A_{i}\right\|^{2}, s=$ $\sum_{i=1}^{m} \beta^{p_{i}}\left\|\Delta A_{i}\right\|\left(2\left\|A_{i}\right\|+\left\|\Delta A_{i}\right\|\right)$. If

$$
\begin{gathered}
0<b<2(\beta-s), \\
b^{2}-4(\beta-s)(s+\|\Delta Q\|) \geq 0,
\end{gathered}
$$

then

$$
\frac{\|\widetilde{X}-X\|}{\|X\|} \leq \varrho \sum_{i=1}^{m}\left\|\Delta A_{i}\right\|+\omega\|\Delta Q\| \triangleq \xi_{1},
$$


where

$$
\begin{aligned}
& \varrho=\frac{2 s}{\sum_{i=1}^{m}\left\|\Delta A_{i}\right\|\left(b+\sqrt{b^{2}-4(\beta-s)(s+\|\Delta Q\|)}\right)}, \\
& \omega=\frac{2}{b+\sqrt{b^{2}-4(\beta-s)(s+\|\Delta Q\|)}} .
\end{aligned}
$$

Proof. Let

$$
\begin{gathered}
\Omega=\left\{\Delta X \in \mathscr{H}^{n \times n}:\left\|X^{-1 / 2} \Delta X X^{-1 / 2}\right\|\right. \\
\left.\leq \varrho \sum_{i=1}^{m}\left\|\Delta A_{i}\right\|+\omega\|\Delta Q\|\right\} .
\end{gathered}
$$

Obviously, $\Omega$ is a nonempty bounded convex closed set. Let

$$
\begin{aligned}
f(\Delta X)= & \sum_{i=1}^{m}\left(\widetilde{A}_{i}^{*}(X+\Delta X)^{p_{i}} \widetilde{A}_{i}-A_{i}^{*} X^{p_{i}} A_{i}\right) \\
& +\Delta Q, \quad \Delta X \in \Omega .
\end{aligned}
$$

Evidently, $f: \Omega \mapsto \mathscr{H}^{n \times n}$ is continuous. We will prove that $f(\Omega) \subseteq \Omega$.

For every $\Delta X \in \Omega$, it follows that $\left\|X^{-1 / 2} \Delta X X^{-1 / 2}\right\| \leq$ $\varrho \sum_{i=1}^{m}\left\|\Delta A_{i}\right\|+\omega\|\Delta Q\|$. Thus

$$
\begin{gathered}
\left(\varrho \sum_{i=1}^{m}\left\|\Delta A_{i}\right\|+\omega\|\Delta Q\|\right) I \\
\geq X^{-1 / 2} \Delta X X^{-1 / 2} \\
\geq\left(-\varrho \sum_{i=1}^{m}\left\|\Delta A_{i}\right\|-\omega\|\Delta Q\|\right) I, \\
\left(1+\varrho \sum_{i=1}^{m}\left\|\Delta A_{i}\right\|+\omega\|\Delta Q\|\right) X \\
\geq X+\Delta X \\
\geq\left(1-\varrho \sum_{i=1}^{m}\left\|\Delta A_{i}\right\|-\omega\|\Delta Q\|\right) X .
\end{gathered}
$$

According to (9), we have

$$
\begin{aligned}
\varrho \sum_{i=1}^{m}\left\|\Delta A_{i}\right\|+\omega\|\Delta Q\| & =\frac{2(\|\Delta Q\|+s)}{b+\sqrt{b^{2}-4(\beta-s)(s+\|\Delta Q\|)}} \\
& \leq \frac{2(\|\Delta Q\|+s)}{b} \leq \frac{b}{2(\beta-s)}<1 .
\end{aligned}
$$

Therefore

$$
\left(1-\varrho \sum_{i=1}^{m}\left\|\Delta A_{i}\right\|-\omega\|\Delta Q\|\right) X>0
$$

From Lemmas 2 and 4, it follows that

$$
\begin{aligned}
& \left\|X^{-1 / 2}\left[\sum_{i=1}^{m} A_{i}^{*}\left((X+\Delta X)^{p_{i}}-X^{p_{i}}\right) A_{i}\right] X^{-1 / 2}\right\| \\
& \leq\left(\left\|X^{-1 / 2} \Delta X X^{-1 / 2}\right\|\right. \\
& \left.\quad+\frac{\left\|X^{-1 / 2} \Delta X X^{-1 / 2}\right\|^{2}}{1-\varrho \sum_{i=1}^{m}\left\|\Delta A_{i}\right\|-\omega\|\Delta Q\|}\right) \\
& \quad \times\left(\sum_{i=1}^{m} p_{i}\left\|X^{p_{i} / 2} A_{i} X^{-1 / 2}\right\|^{2}\right) \\
& \leq\left(\left\|X^{-1 / 2} \Delta X X^{-1 / 2}\right\|+\frac{\left\|X^{-1 / 2} \Delta X X^{-1 / 2}\right\|^{2}}{1-\varrho \sum_{i=1}^{m}\left\|\Delta A_{i}\right\|-\omega\|\Delta Q\|}\right) \\
& \quad \times\left(\sum_{i=1}^{m} \frac{p_{i}}{\beta^{1-p_{i}}\left\|A_{i}\right\|^{2}}\right) .
\end{aligned}
$$

Therefore

$$
\begin{aligned}
& \left\|X^{-1 / 2} f(\Delta X) X^{-1 / 2}\right\| \\
& =\| X^{-1 / 2}\left[\sum_{i=1}^{m} \widetilde{A}_{i}^{*}(X+\Delta X)^{p_{i}} \widetilde{A}_{i}-A_{i}^{*} X^{p_{i}} A_{i}\right] \\
& \quad \times X^{-1 / 2}+X^{-1 / 2} \Delta Q X^{-1 / 2} \| \\
& \leq\left\|\sum_{i=1}^{m} X^{-1 / 2} A_{i}^{*}\left((X+\Delta X)^{p_{i}}-X^{p_{i}}\right) A_{i} X^{-1 / 2}\right\| \\
& +\left\|X^{-1 / 2} \Delta Q X^{-1 / 2}\right\| \\
& +\| \sum_{i=1}^{m} X^{-1 / 2}\left[\Delta A_{i}^{*}(X+\Delta X)^{p_{i}}\left(A_{i}+\Delta A_{i}\right)\right. \\
& \left.\quad+A_{i}^{*}(X+\Delta X)^{p_{i}} \Delta A_{i}\right] X^{-1 / 2} \| \\
& \leq\left(\left\|X^{-1 / 2} \Delta X X^{-1 / 2}\right\|+\frac{\left\|X^{-1 / 2} \Delta X X^{-1 / 2}\right\|^{2}}{1-\varrho \sum_{i=1}^{m}\left\|\Delta A_{i}\right\|-\omega\|\Delta Q\|}\right) \\
& \quad+\left(\sum_{i=1}^{m} \frac{p_{i}}{\left.\beta^{1-p_{i}}\left\|A_{i}\right\|^{2}\right)}\right. \\
& +\sum_{i=1}^{m} \frac{\left\|\Delta A_{i}\right\|\left(2\left\|A_{i}\right\|+\left\|\Delta A_{i}\right\|\right)}{\beta^{1-p_{i}}}
\end{aligned}
$$




$$
\begin{aligned}
& \quad \times\left(1+\varrho \sum_{i=1}^{m}\left\|\Delta A_{i}\right\|+\omega\|\Delta Q\|\right)+\frac{\|\Delta Q\|}{\beta} \\
& \leq\left(\xi_{1}+\frac{\xi_{1}^{2}}{1-\xi_{1}}\right)\left(\sum_{i=1}^{m} \frac{p_{i}}{\beta^{1-p_{i}}}\left\|A_{i}\right\|^{2}\right) \\
& +\frac{s}{\beta}\left(1+\xi_{1}\right)+\frac{\|\Delta Q\|}{\beta} \\
& =\xi_{1} .
\end{aligned}
$$

That is, $f(\Omega) \subseteq \Omega$. By Brouwer's fixed point theorem, there exists a $\Delta X \in \Omega$ such that $f(\Delta X)=\Delta X$. Moreover, by Lemma 3, we know that $X$ and $\widetilde{X}$ are the unique solutions to (1) and (7), respectively. Then

$$
\begin{aligned}
\frac{\|\widetilde{X}-X\|}{\|X\|} & =\frac{\|\Delta X\|}{\|X\|}=\frac{\left\|X^{1 / 2}\left(X^{-1 / 2} \Delta X X^{-1 / 2}\right) X^{1 / 2}\right\|}{\|X\|} \\
& \leq\left\|X^{-1 / 2} \Delta X X^{-1 / 2}\right\| \leq \varrho \sum_{i=1}^{m}\left\|\Delta A_{i}\right\|+\omega\|\Delta Q\| .
\end{aligned}
$$

Remark 6. According to

$$
\begin{aligned}
\varrho \sum_{i=1}^{m} \| & \Delta A_{i}\|+\omega\| \Delta Q \| \\
& =\frac{2\left(\sum_{i=1}^{m}\left\|\Delta A_{i}\right\|\left(2\left\|A_{i}\right\|+\left\|\Delta A_{i}\right\|\right)+\|\Delta Q\|\right)}{b+\sqrt{b^{2}-4(\beta-s)(s+\|\Delta Q\|)}},
\end{aligned}
$$

we get $\varrho \sum_{i=1}^{m}\left\|\Delta A_{i}\right\|+\omega\|\Delta Q\| \rightarrow 0$ for $\|\Delta Q\| \rightarrow 0$ and $\left\|\Delta A_{i}\right\| \rightarrow 0(i=1,2, \ldots, m)$. Therefore (1) is well posed.

Next, a sharper perturbation estimate is derived.

Subtracting (1) from (7), we have

$$
\begin{aligned}
\Delta X+\sum_{i=1}^{m} \frac{\sin p_{i} \pi}{\pi} \int_{0}^{\infty}\left[(\lambda I+X)^{-1} X^{1 / 2} A_{i}\right]^{*} & \\
& \times \Delta X\left[(\lambda I+X)^{-1} X^{1 / 2} A_{i}\right] \lambda^{p_{i}-1} d \lambda \\
& =E+h(\Delta X),
\end{aligned}
$$

where

$$
\begin{gathered}
B_{i}=X^{p_{i}} A_{i}, \quad i=1,2, \ldots, m, \\
E=\sum_{i=1}^{m}\left(B_{i}^{*} \Delta A_{i}+\Delta A_{i}^{*} B_{i}\right)+\sum_{i=1}^{m} \Delta A_{i}^{*} X^{p_{i}} \Delta A_{i}+\Delta Q
\end{gathered}
$$

$$
\begin{gathered}
Z_{i}(\Delta X)=\frac{\sin p_{i} \pi}{\pi} \int_{0}^{\infty} X^{1 / 2}(\lambda I+X)^{-1} \Delta X(\lambda I+X+\Delta X)^{-1} \\
\quad \times \Delta X(\lambda I+X)^{-1} X^{1 / 2} \lambda^{p_{i}-1} d \lambda \\
V_{i}(\Delta X)=\frac{\sin p_{i} \pi}{\pi} \int_{0}^{\infty} X^{1 / 2}(\lambda I+X)^{-1} \Delta X \\
\quad \times(\lambda I+X+\Delta X)^{-1} X^{1 / 2} \lambda^{p_{i}-1} d \lambda \\
h(\Delta X)=\sum_{i=1}^{m}\left[A_{i}^{*} Z_{i}(\Delta X) A_{i}-\widetilde{A}_{i}^{*} V_{i}(\Delta X) \Delta A_{i}\right. \\
\left.-\Delta A_{i}^{*} V_{i}(\Delta X) A_{i}\right] .
\end{gathered}
$$

Lemma 7. If $\sum_{i=1}^{m}\left(\left\|A_{i}\right\|^{2} / \beta^{1-p_{i}}\right)<1$, then the linear operator $\mathbf{L}: \mathscr{H}^{n \times n} \rightarrow \mathscr{H}^{n \times n}$ defined by

$$
\begin{aligned}
& \mathbf{L} W=W+ \sum_{i=1}^{m} \frac{\sin p_{i} \pi}{\pi} \\
& \times \int_{0}^{\infty}\left[(\lambda I+X)^{-1} X^{1 / 2} A_{i}\right]^{*} \\
& \quad \times W\left[(\lambda I+X)^{-1} X^{1 / 2} A_{i}\right] \\
& \quad \times \lambda^{p_{i}-1} d \lambda, \quad W \in \mathscr{H}^{n \times n}
\end{aligned}
$$

is invertible.

Proof. It suffices to show that the following equation:

$$
\mathbf{L} W=V
$$

has a unique solution for every $V \in \mathscr{H}^{n \times n}$. Define the operator $\mathbf{M}: \mathscr{H}^{n \times n} \rightarrow \mathscr{H}^{n \times n}$ by

$$
\begin{aligned}
& \mathbf{M} Z=\sum_{i=1}^{m} \frac{\sin p_{i} \pi}{\pi} \\
& \times \int_{0}^{\infty} X^{-1 / 2} A_{i}^{*} X^{1 / 2} \\
& \quad \times(\lambda I+X)^{-1} X^{1 / 2} Z X^{1 / 2} \\
& \quad \times(\lambda I+X)^{-1} X^{1 / 2} A_{i} X^{-1 / 2} \\
& \quad \times \lambda^{p_{i}-1} d \lambda, \quad Z \in \mathscr{H}^{n \times n} .
\end{aligned}
$$

Let $Y=X^{-1 / 2} W X^{-1 / 2}$. Thus (21) is equivalent to

$$
Y+\mathbf{M} Y=X^{-1 / 2} V X^{-1 / 2}
$$


According to Lemma 2, we have

$$
\begin{gathered}
\|\mathbf{M Y}\| \leq \sum_{i=1}^{m} \| \frac{\sin p_{i} \pi}{\pi} \\
\quad \times \int_{0}^{\infty} X^{-1 / 2} A_{i}^{*} X^{1 / 2} \\
\times(\lambda I+X)^{-1} X(\lambda I+X)^{-1} \\
\quad \times X^{1 / 2} A_{i} X^{-1 / 2} \lambda^{p_{i}-1} d \lambda\|\| Y \| \\
\leq \sum_{i=1}^{m}\left(1-p_{i}\right)\left\|X^{p_{i} / 2} A_{i} X^{-1 / 2}\right\|^{2}\|Y\| \\
\leq \sum_{i=1}^{m} \frac{\left\|A_{i}\right\|^{2}}{\beta^{1-p_{i}}}\|Y\|<\|Y\|,
\end{gathered}
$$

which implies that $\|\mathbf{M}\|<1$ and $I+\mathbf{M}$ is invertible. Therefore, the operator $\mathbf{L}$ is invertible.

Furthermore, we define operators $\mathbf{P}_{i}: \mathscr{C}^{n \times n} \rightarrow \mathscr{H}^{n \times n}$ by

$$
\mathbf{P}_{i} Z_{i}=\mathbf{L}^{-1}\left(B_{i}^{*} Z_{i}+Z_{i}^{*} B_{i}\right), \quad Z_{i} \in \mathscr{C}^{n \times n}, i=1,2, \ldots, m .
$$

Thus, we can rewrite (21) as

$$
\begin{aligned}
\Delta X= & \mathbf{L}^{-1} \Delta Q \\
& +\sum_{i=1}^{m} \mathbf{P}_{i} \Delta A_{i}+\mathbf{L}^{-1}\left(\sum_{i=1}^{m} \Delta A_{i}^{*} X^{p_{i}} \Delta A_{i}\right) \\
& +\mathbf{L}^{-1}(h(\Delta X)) .
\end{aligned}
$$

Define

$$
\begin{aligned}
\left\|\mathbf{L}^{-1}\right\| & =\max _{\substack{W \in \mathscr{P} \mathscr{C}^{n \times n} \\
\|W\|=1}}\left\|\mathbf{L}^{-1} W\right\|, \\
\left\|\mathbf{P}_{i}\right\| & =\max _{\substack{Z \in \mathscr{C}^{n \times n} \\
\|Z\|=1}}\left\|\mathbf{P}_{i} Z\right\|, \quad i=1,2, \ldots, m .
\end{aligned}
$$

Now we denote that

$$
\begin{gathered}
l=\left\|\mathbf{L}^{-1}\right\|^{-1}, \quad \zeta=\left\|X^{-1}\right\|, \\
\xi_{i}=\left\|X^{p_{i}}\right\|, \quad n_{i}=\left\|\mathbf{P}_{i}\right\| \\
\theta=\frac{\zeta^{2}}{l} \sum_{i=1}^{m} \xi_{i}\left\|A_{i}\right\|^{2} \\
i=1,2, \ldots, m
\end{gathered}
$$

$$
\begin{gathered}
\epsilon=\frac{1}{l}\|\Delta Q\|+\sum_{i=1}^{m}\left(n_{i}\left\|\Delta A_{i}\right\|+\frac{\xi_{i}}{l}\left\|\Delta A_{i}\right\|^{2}\right), \\
\sigma=\frac{\zeta}{l} \sum_{i=1}^{m} \xi_{i}\left(2\left\|A_{i}\right\|+\left\|\Delta A_{i}\right\|\right)\left\|\Delta A_{i}\right\| .
\end{gathered}
$$

Theorem 8. Suppose that $X$ and $\widetilde{X}$ are the solutions of (1) and (7), respectively. If $\sum_{i=1}^{m}\left\|A_{i}\right\|^{2} / \beta^{1-p_{i}}<1$,

$$
\begin{gathered}
\sigma<1, \\
\epsilon<\frac{(1-\sigma)^{2}}{\zeta+\sigma \zeta+2 \theta+2 \sqrt{((\zeta+\theta)(\sigma \zeta+\theta))}}
\end{gathered}
$$

then

$$
\|\widetilde{X}-X\| \leq \frac{2 \epsilon}{1+\epsilon \zeta-\sigma+\sqrt{(1+\zeta \epsilon-\sigma)^{2}-4 \epsilon(\zeta+\theta)}} \equiv \nu .
$$

Proof. Let

$$
\begin{aligned}
f(\Delta X)= & \mathbf{L}^{-1} \Delta Q+\sum_{i=1}^{m} \mathbf{P}_{i} \Delta A_{i} \\
& +\mathbf{L}^{-1}\left(\sum_{i=1}^{m} \Delta A_{i}^{*} X^{p_{i}} \Delta A_{i}\right)+\mathbf{L}^{-1}(h(\Delta X)) .
\end{aligned}
$$

Obviously, $f: \mathscr{H}^{n \times n} \rightarrow \mathscr{H}^{n \times n}$ is continuous. The condition (32) ensures that the quadratic equation $(\zeta+\theta) x^{2}-(1+\zeta \epsilon-$ $\sigma) x+\epsilon=0$ with respect to the variable $x$ has two positive real roots. The smaller one is

$$
v=\frac{2 \epsilon}{1+\epsilon \zeta-\sigma+\sqrt{(1+\zeta \epsilon-\sigma)^{2}-4 \epsilon(\zeta+\theta)}} .
$$

Define $\Omega=\left\{\Delta X \in \mathscr{H}^{n \times n}:\|\Delta X\| \leq \nu\right\}$. Then for any $\Delta X \in \Omega$, by (32), we have

$$
\begin{aligned}
\left\|X^{-1} \Delta X\right\| & \leq\left\|X^{-1}\right\|\|\Delta X\| \leq \zeta \nu \\
& \leq \zeta \cdot \frac{2 \epsilon}{1+\zeta \epsilon-\sigma}=1+\frac{\zeta \epsilon+\sigma-1}{1+\zeta \epsilon-\sigma} \\
& \leq 1+\frac{-2(1-\sigma)(\zeta \sigma+\theta)}{(\zeta+\sigma \zeta+2 \theta)(1+\zeta \epsilon-\sigma)}<1 .
\end{aligned}
$$

It follows that $I-X^{-1} \Delta X$ is nonsingular and

$$
\left\|I-X^{-1} \Delta X\right\| \leq \frac{1}{1-\left\|X^{-1} \Delta X\right\|} \leq \frac{1}{1-\zeta\|\Delta X\|} .
$$


Using (22) and Lemma 2, we have

$$
\begin{aligned}
& \left\|Z_{i}(\Delta X)\right\| \leq\left(1-p_{i}\right)\|\Delta X\|^{2}\left\|X^{-1}\right\|^{2} \\
& \times\left\|\left(I+X^{-1} \Delta X\right)^{-1}\right\|\left\|X^{p_{i}}\right\| \\
& \leq \xi_{i} \zeta^{2} \frac{\|\Delta X\|^{2}}{1-\zeta\|\Delta X\|} \\
& \left\|V_{i}(\Delta X)\right\| \leq\left\|X^{p_{i}}\right\|\|\Delta X\|\left\|X^{-1}\right\|\left\|\left(I+X^{-1} \Delta X\right)^{-1}\right\| \\
& \leq \xi_{i} \zeta \frac{\|\Delta X\|}{1-\zeta\|\Delta X\|}, \\
& \|h(\Delta X)\| \leq \sum_{i=1}^{m}\left(\left\|A_{i}\right\|^{2}\left\|Z_{i}(\Delta X)\right\|\right. \\
& +\left(2\left\|A_{i}\right\|+\left\|\Delta A_{i}\right\|\right) \\
& \left.\times\left\|\Delta A_{i}\right\|\left\|V_{i}(\Delta X)\right\|\right) \\
& \leq \sum_{i=1}^{m}\left(\xi_{i} \zeta^{2}\left\|A_{i}\right\|^{2} \frac{\|\Delta X\|^{2}}{1-\zeta\|\Delta X\|}\right. \\
& +\left(2\left\|A_{i}\right\|+\left\|\Delta A_{i}\right\|\right) \\
& \left.\times\left\|\Delta A_{i}\right\| \xi_{i} \zeta \frac{\|\Delta X\|}{1-\zeta\|\Delta X\|}\right) .
\end{aligned}
$$

Noting (31) and (34), it follows that

$$
\begin{aligned}
\|f(\Delta X)\| \leq & \frac{1}{l}\|\Delta Q\|+\sum_{i=1}^{m}\left(n_{i}\left\|\Delta A_{i}\right\|+\frac{\zeta_{i}}{l}\left\|\Delta A_{i}\right\|^{2}\right) \\
& +\frac{1}{l}\|h(\Delta X)\| \\
\leq & \epsilon+\frac{\sigma\|\Delta X\|}{1-\zeta\|\Delta X\|}+\frac{\theta\|\Delta X\|^{2}}{1-\zeta\|\Delta X\|} \\
\leq & \epsilon+\frac{\sigma \nu}{1-\zeta v}+\frac{\theta v^{2}}{1-\zeta \nu}=\nu
\end{aligned}
$$

for $\Delta X \in \Omega$. That is, $f(\Omega) \subseteq \Omega$. According to Schauder fixed point theorem, there exists $\Delta X_{*} \in \Omega$ such that $f\left(\Delta X_{*}\right)=X_{*}$. It follows that $X+\Delta X_{*}$ is a Hermitian solution of (7). By Lemma 3, we know that the solution of (7) is unique. Then $\left\|\Delta X_{*}\right\|=\|\widetilde{X}-X\| \leq \nu$.

Remark 9. From Theorem 8, we get the first order perturbation bound for the solution as follows:

$$
\begin{aligned}
\|\widetilde{X}-X\| \leq & \frac{1}{l}\|\Delta Q\|+\sum_{i=1}^{m} n_{i}\left\|\Delta A_{i}\right\| \\
& +O\left(\left\|\left(\Delta A_{1}, \Delta A_{2}, \ldots, \Delta A_{m}, \Delta Q\right)\right\|_{F}^{2}\right),
\end{aligned}
$$

Combining this with (29) gives

$$
\begin{aligned}
& \Delta X=\mathbf{L}^{-1} \Delta Q+\mathbf{L}^{-1} \sum_{i=1}^{m}\left(B_{i}^{*} \Delta A_{i}+\Delta A_{i}^{*} B_{i}\right) \\
& +O\left(\left\|\left(\Delta A_{1}, \Delta A_{2}, \ldots, \Delta A_{m}, \Delta Q\right)\right\|_{F}^{2}\right) . \\
& \text { as }\left(\Delta A_{1}, \Delta A_{2}, \ldots, \Delta A_{m}, \Delta Q\right) \rightarrow 0 \text {. }
\end{aligned}
$$

\section{Backward Error}

In this section, a backward error of an approximate solution for the unique solution to (1) is obtained.

Theorem 10. Let $\widetilde{X}>0$ be an approximation to the solution $X$ of (1). If $\Sigma=\sum_{i=1}^{m} p_{i}\left\|\widetilde{X}^{p_{i} / 2} A_{i} \widetilde{X}^{-1 / 2}\right\|^{2}<1$ and the residual $R(\widetilde{X}) \equiv Q+\sum_{i=1}^{m} A_{i}^{*} \widetilde{X}^{p_{i}} A_{i}-\widetilde{X}$ satisfies

$$
\|R(\widetilde{X})\|<\frac{\theta_{1}}{2\left\|\widetilde{X}^{-1}\right\|} \min \left\{1, \frac{\theta_{1}}{2}\right\},
$$

where $\theta_{1} \equiv 1+\left\|\widetilde{X}^{-1}\right\|\|R(\widetilde{X})\|-\Sigma>0$,

then

$$
\begin{gathered}
\qquad\|\widetilde{X}-X\| \leq \mu\|R(\widetilde{X})\|, \\
\text { where } \mu=\frac{2\|\widetilde{X}\|\left\|\widetilde{X}^{-1}\right\|}{\theta_{1}+\sqrt{\theta_{1}^{2}-4\left\|\widetilde{X}^{-1}\right\|\|R(\widetilde{X})\|}} .
\end{gathered}
$$

Proof. Let

$$
\Psi=\left\{\Delta X \in \mathscr{H}^{n \times n}:\left\|\widetilde{X}^{-1 / 2} \Delta X \widetilde{X}^{-1 / 2}\right\| \leq \theta_{2}\|R(\widetilde{X})\|\right\},
$$

where $\theta_{2}=\mu /\|\widetilde{X}\|$. Obviously, $\Psi$ is a nonempty bounded convex closed set. Let

$$
g(\Delta X)=\sum_{i=1}^{m} A_{i}^{*}\left[(\widetilde{X}+\Delta X)^{p_{i}}-\widetilde{X}^{p_{i}}\right] A_{i}+R(\widetilde{X}) .
$$

Evidently $g$ : $\Psi \mapsto \mathscr{H}^{n \times n}$ is continuous. We will prove that $g(\Psi) \subseteq \Psi$. For every $\Delta X \in \Psi$, we have

$$
\left\|\widetilde{X}^{-1 / 2} \Delta X \widetilde{X}^{-1 / 2}\right\| \leq \theta_{2}\|R(\widetilde{X})\| .
$$

Hence

$$
\widetilde{X}^{-1 / 2} \Delta X \widetilde{X}^{-1 / 2} \geq-\theta_{2}\|R(\widetilde{X})\| I
$$

That is,

$$
\widetilde{X}+\Delta X \geq\left(1-\theta_{2}\|R(\widetilde{X})\|\right) \widetilde{X}
$$

Using (43), one sees that

$$
\begin{aligned}
\theta_{2}\|R(\widetilde{X})\| & =\frac{2\left\|\widetilde{X}^{-1}\right\|\|R(\widetilde{X})\|}{\theta_{1}+\sqrt{\theta_{1}^{2}-4\left\|\widetilde{X}^{-1}\right\|\|R(\widetilde{X})\|}} \\
& <\frac{2\left\|\widetilde{X}^{-1}\right\|\|R(\widetilde{X})\|}{\theta_{1}}<1 .
\end{aligned}
$$

Therefore $\left(1-\theta_{2}\|R(\widetilde{X})\|\right) \widetilde{X}>0$. 
According to (17), we obtain

$$
\begin{aligned}
\left\|\widetilde{X}^{-1 / 2} g(\Delta X) \widetilde{X}^{-1 / 2}\right\| & \\
\leq & \left(\left\|\widetilde{X}^{-1 / 2} \Delta X \widetilde{X}^{-1 / 2}\right\|+\frac{\left\|\widetilde{X}^{-1 / 2} \Delta X \widetilde{X}^{-1 / 2}\right\|^{2}}{1-\theta_{2}\|R(\widetilde{X})\|}\right) \Sigma \\
& +\left\|\widetilde{X}^{-1 / 2} R(\widetilde{X}) \widetilde{X}^{-1 / 2}\right\| \\
\leq & \left(\theta_{2}\|R(\widetilde{X})\|+\frac{\left(\theta_{2}\|R(\widetilde{X})\|\right)^{2}}{1-\theta_{2}\|R(\widetilde{X})\|}\right) \Sigma+\left\|\widetilde{X}^{-1}\right\|\|R(\widetilde{X})\| \\
= & \theta_{2}\|R(\widetilde{X})\|,
\end{aligned}
$$

for $\Delta X \in \Psi$. That is, $g(\Psi) \subseteq \Psi$. By Brouwer's fixed point theorem, there exists a $\Delta X \in \Psi$ such that $g(\Delta X)=\Delta X$. Hence $\widetilde{X}+\Delta X$ is a solution of (1). Moreover, by Lemma 3 , we know that the solution $X$ of (1) is unique. Then

$$
\begin{aligned}
\|\widetilde{X}-X\| & =\|\Delta X\| \leq\|\widetilde{X}\|\left\|\widetilde{X}^{-1 / 2} \Delta X \widetilde{X}^{-1 / 2}\right\| \\
& =\theta_{2}\|\widetilde{X}\|\|R(\widetilde{X})\|=\mu\|R(\widetilde{X})\| .
\end{aligned}
$$

\section{Condition Number}

In this section, we apply the theory of condition number developed by Rice [36] to study condition numbers of the unique solution to (1).
5.1. The Complex Case. Suppose that $X$ and $\widetilde{X}$ are the solutions of the matrix equations (1) and (7), respectively. Let $\Delta A_{i}=\widetilde{A}_{i}-A_{i}, \Delta Q=\widetilde{Q}-Q$ and $\Delta X=\widetilde{X}-X$. Using Theorem 8 and Remark 9 , we have

$$
\begin{aligned}
\Delta X= & \widetilde{X}-X=\mathbf{L}^{-1} \Delta Q \\
& +\mathbf{L}^{-1} \sum_{i=1}^{m}\left(B_{i}^{*} \Delta A_{i}+\Delta A_{i}^{*} B_{i}\right) \\
& +O\left(\left\|\left(\Delta A_{1}, \Delta A_{2}, \ldots, \Delta A_{m}, \Delta Q\right)\right\|_{F}^{2}\right),
\end{aligned}
$$

as $\left(\Delta A_{1}, \Delta A_{2}, \ldots, \Delta A_{m}, \Delta Q\right) \rightarrow 0$.

By the theory of condition number developed by Rice [36], we define the condition number of the Hermitian positive definite solution $X$ to (1) by

$$
c(X)=\lim _{\delta \rightarrow 0} \sup _{\left\|\left(\Delta A_{1} / \eta_{1}, \Delta A_{2} / \eta_{2}, \ldots, \Delta A_{m} / \eta_{m}, \Delta Q / \rho\right)\right\|_{F} \leq \delta} \frac{\|\Delta X\|_{F}}{\xi \delta},
$$

where $\xi, \rho$, and $\eta_{i}, i=1,2, \ldots, m$, are positive parameters. Taking $\xi=\eta_{i}=\rho=1$ in (54) gives the absolute condition number $c_{\text {abs }}(X)$, and taking $\xi=\|X\|_{F}, \eta_{i}=\left\|A_{i}\right\|_{F}$, and $\rho=$ $\|Q\|_{F}$ in (54) gives the relative condition number $c_{\text {rel }}(X)$.

Substituting (53) into (54), we get

$$
\begin{gathered}
c(X)=\frac{1}{\xi} \max _{\substack{\left(\Delta A_{1} / \eta_{1}, \Delta A_{2} / \eta_{2}, \ldots, \Delta A_{m} / \eta_{m}, \Delta \mathrm{Q} / \rho\right) \neq 0 \\
\Delta A_{i} \in \mathscr{C}^{n \times n}, \Delta \mathrm{Q} \in \mathscr{\mathscr { H } ^ { n \times n }}}} \frac{\left\|\mathbf{L}^{-1}\left(\Delta Q+\sum_{i=1}^{m}\left(B_{i}^{*} \Delta A_{i}+\Delta A_{i}^{*} B_{i}\right)\right)\right\|_{F}}{\left\|\left(\Delta A_{1} / \eta_{1}, \Delta A_{2} / \eta_{2}, \ldots, \Delta A_{m} / \eta_{m}, \Delta Q / \rho\right)\right\|_{F}} \\
=\frac{1}{\xi} \max _{\substack{\left(E_{1}, E_{2}, \ldots, E_{m}, H\right) \neq 0 \\
E_{i} \in \mathscr{C}^{n \times n}, H \in \mathscr{H}^{n \times n}}} \frac{\left\|\mathbf{L}^{-1}\left(\rho H+\sum_{i=1}^{m} \eta_{i}\left(B_{i}^{*} E_{i}+E_{i}^{*} B_{i}\right)\right)\right\|_{F}}{\left\|\left(E_{1}, E_{2}, \ldots, E_{m}, H\right)\right\|_{F}} .
\end{gathered}
$$

Let $L$ be the matrix representation of the linear operator $\mathbf{L}$. Then it is easy to see that

$$
\begin{aligned}
L=I+\sum_{i=1}^{m} \frac{\sin p_{i} \pi}{\pi} \int_{0}^{\infty}[ & \left.(\lambda I+X)^{-1} X^{1 / 2} A_{i}\right]^{T} \\
\otimes & {\left[(\lambda I+X)^{-1} X^{1 / 2} A_{i}\right]^{*} } \\
& \times \lambda^{p_{i}-1} d \lambda .
\end{aligned}
$$

Let

$$
\begin{gathered}
L^{-1}=S+\mathbf{i} \Sigma, \\
L^{-1}\left(I \otimes B_{i}^{*}\right)=L^{-1}\left(I \otimes\left(X^{p_{i}} A_{i}\right)^{*}\right)=U_{i 1}+\mathbf{i} \Omega_{i 1}, \\
L^{-1}\left(B_{i}^{T} \otimes I\right) \Pi=L^{-1}\left(\left(X^{p_{i}} A_{i}\right)^{T} \otimes I\right) \Pi=U_{i 2}+\mathbf{i} \Omega_{i 2}, \\
S_{c}=\left[\begin{array}{cc}
S & -\Sigma \\
\Sigma & S
\end{array}\right], \\
U_{i}=\left[\begin{array}{ll}
U_{i 1}+U_{i 2} & \Omega_{i 2}-\Omega_{i 1} \\
\Omega_{i 1}+\Omega_{i 2} & U_{i 1}-U_{i 2}
\end{array}\right], \quad i=1,2, \ldots, m,
\end{gathered}
$$


$\operatorname{vec} H=x+\mathbf{i} y, \quad \operatorname{vec} E_{i}=a_{i}+\mathbf{i} b_{i}, \quad g=\left(x^{T}, y^{T}, a_{1}^{T}\right.$, $\left.b_{1}^{T}, \ldots, a_{m}^{T}, b_{m}^{T}\right)^{T}, M=\left(E_{1}, E_{2}, \ldots, E_{m}, H\right)$, where $x, y, a_{i}, b_{i} \in$ $\mathscr{R}^{n^{2}}, S, \Sigma, U_{i 1}, U_{i 2}, \Omega_{i 1}, \Omega_{i 2} \in \mathscr{R}^{n^{2} \times n^{2}}, i=1,2, \ldots, m$, and $\Pi$ is the vec-permutation matrix, such that

$$
\operatorname{vec} A^{T}=\Pi \operatorname{vec} A
$$

Then we obtain that

$$
\begin{aligned}
& c(X)=\frac{1}{\xi} \max _{M \neq 0} \frac{\left\|\mathbf{L}^{-1}\left(\rho H+\sum_{i=1}^{m} \eta_{i}\left(B_{i}^{*} E_{i}+E_{i}^{*} B_{i}\right)\right)\right\|_{F}}{\left\|\left(E_{1}, E_{2}, \ldots, E_{m}, H\right)\right\|_{F}} \\
& =\frac{1}{\xi} \max _{M \neq 0}\left(\| \rho L^{-1} \operatorname{vec} H\right. \\
& +\sum_{i=1}^{m} \eta_{i} L^{-1}\left(\left(I \otimes B_{i}^{*}\right) \operatorname{vec} E_{i}\right. \\
& \left.\left.+\left(B_{i}^{T} \otimes I\right) \operatorname{vec} E_{i}^{*}\right) \|\right) \\
& \times\left(\left\|\operatorname{vec}\left(E_{1}, E_{2}, \ldots, E_{m}, H\right)\right\|\right)^{-1} \\
& =\frac{1}{\xi} \max _{M \neq 0}(\| \rho(S+\mathbf{i} \Sigma)(x+\mathbf{i} y) \\
& +\sum_{i=1}^{m} \eta_{i}\left[\left(U_{i 1}+\mathbf{i} \Omega_{i 1}\right)\left(a_{i}+\mathbf{i} b_{i}\right)\right. \\
& \left.\left.+\left(U_{i 2}+\mathbf{i} \Omega_{i 2}\right)\left(a_{i}-\mathbf{i} b_{i}\right)\right] \|\right) \\
& \times\left(\left\|\operatorname{vec}\left(E_{1}, E_{2}, \ldots, E_{m}, H\right)\right\|\right)^{-1} \\
& =\frac{1}{\xi} \max _{g \neq 0} \frac{\left\|\left(\rho S_{c}, \eta_{1} U_{1}, \eta_{2} U_{2}, \ldots, \eta_{m} U_{m}\right) g\right\|}{\|g\|} \\
& =\frac{1}{\xi}\left\|\left(\rho S_{c}, \eta_{1} U_{1}, \eta_{2} U_{2}, \ldots, \eta_{m} U_{m}\right)\right\|, \\
& E_{i} \in \mathscr{C}^{n \times n}, \quad H \in \mathscr{H}^{n \times n} .
\end{aligned}
$$

Then we have the following theorem.

Theorem 11. If $\sum_{i=1}^{m}\left\|A_{i}\right\|^{2} / \beta^{1-p_{i}}<1$, then the condition number $c(X)$ defined by (54) has the explicit expression

$$
c(X)=\frac{1}{\xi}\left\|\left(\rho S_{c}, \eta_{1} U_{1}, \eta_{2} U_{2}, \ldots, \eta_{m} U_{m}\right)\right\|,
$$

where the matrices $S_{c}$ and $U_{i}$ are defined as in (57).
Remark 12. From (60) we have the relative condition number

$$
c_{\text {rel }}(X)=\frac{\left\|\left(\|Q\|_{F} S_{c},\left\|A_{1}\right\|_{F} U_{1},\left\|A_{2}\right\|_{F} U_{2}, \ldots,\left\|A_{m}\right\|_{\mathrm{F}} U_{m}\right)\right\|}{\|X\|_{F}} .
$$

5.2. The Real Case. In this subsection we consider the real case. That is, all the coefficient matrices $A_{i}, Q$ of (1) are real. In such a case the corresponding solution $X$ is also real. Completely similar arguments as Theorem 11 give the following theorem.

Theorem 13. Let $A_{i}, Q$ be real and let $c(X)$ be the condition number defined by (54). If $\sum_{i=1}^{m}\left\|A_{i}\right\|^{2} / \beta^{1-p_{i}}<1$, then $c(X)$ has the explicit expression

$$
c(X)=\frac{1}{\xi}\left\|\left(\rho S_{r}, \eta_{1} U_{1}, \eta_{2} U_{2}, \ldots, \eta_{m} U_{m}\right)\right\|,
$$

where

$$
\begin{gathered}
S_{r}=\left(I+\sum_{i=1}^{m} \frac{\sin p_{i} \pi}{\pi} \int_{0}^{\infty}\left[(\lambda I+X)^{-1} X^{1 / 2} A_{i}\right]^{T}\right. \\
\left.\otimes\left[(\lambda I+X)^{-1} X^{1 / 2} A_{i}\right]^{T} \lambda^{p_{i}-1} d \lambda\right)^{-1} \\
U_{i}=S_{r}\left[I \otimes\left(A_{i}^{T} X^{p_{i}}\right)+\left(\left(A_{i}^{T} X^{p_{i}}\right) \otimes I\right) \Pi\right] \\
i=1,2, \ldots, m .
\end{gathered}
$$

Remark 14. In the real case the relative condition number is given by

$$
c_{\text {rel }}(X)=\frac{\left\|\left(\|Q\|_{F} S_{r},\left\|A_{1}\right\|_{F} U_{1},\left\|A_{2}\right\|_{F} U_{2}, \ldots,\left\|A_{m}\right\|_{F} U_{m}\right)\right\|}{\|X\|_{F}} .
$$

\section{Numerical Examples}

To illustrate the results of the previous sections, in this section three simple examples are given, which were carried out using MATLAB 7.1. For the stopping criterion we take $\varepsilon_{k+1}(X)=$ $\left\|X_{k}-\sum_{i=1}^{m} A_{i}^{*} X_{k}^{p_{i}} A_{i}-Q\right\|<1.0 e-10$.

Example 15. We consider the matrix equation

$$
X-A_{1}^{*} X^{1 / 2} A_{1}-A_{2}^{*} X^{1 / 3} A_{2}=I,
$$

with

$$
\begin{aligned}
A_{1} & =\frac{(1 / 3)+2 \times 10^{-2}}{\|A\|} A, \\
A_{2} & =\frac{(1 / 6)+3 \times 10^{-2}}{\|A\|} A, \\
A & =\left(\begin{array}{cc}
0 & 0.95 \\
0 & 1
\end{array}\right) .
\end{aligned}
$$


TABLE 1: Assumptions check for Example 15 with different values of $j$.

\begin{tabular}{lcccc}
\hline$j$ & 4 & 5 & 6 & 7 \\
\hline ass $_{1}$ & 1.0749 & 1.0753 & 1.0753 & 1.0753 \\
ass $_{2}$ & 0.9248 & 0.9247 & 0.9247 & 0.9247 \\
ass $_{3}$ & 0.8543 & 0.8550 & 0.8550 & 0.8550 \\
ass $_{4}$ & 0.9999 & 1.0000 & 1.0000 & 1.0000 \\
ass $_{5}$ & 0.7645 & 0.7648 & 0.7648 & 0.7648 \\
\hline
\end{tabular}

Suppose that the coefficient matrices $A_{1}$ and $A_{2}$ are perturbed to $\widetilde{A}_{i}=A_{i}+\Delta A_{i}, i=1,2$, where

$$
\begin{aligned}
& \Delta A_{1}=\frac{10^{-j}}{\left\|C^{T}+C\right\|}\left(C^{T}+C\right), \\
& \Delta A_{2}=\frac{3 \times 10^{-j-1}}{\left\|C^{T}+C\right\|}\left(C^{T}+C\right),
\end{aligned}
$$

and $C$ is a random matrix generated by MATLAB function randn.

We now consider the corresponding perturbation bounds for the solution $X$ in Theorems 5 and 8 .

The assumptions in Theorem 5 are

$$
\begin{aligned}
& \operatorname{ass}_{1}=2(\beta-s)-b>0, \\
& \operatorname{ass}_{2}=\beta+\|\Delta Q\|-\sum_{i=1}^{m} p_{i} \beta^{p_{i}}\left\|A_{i}\right\|^{2}>0, \\
& \operatorname{ass}_{3}=b^{2}-4(\beta-s)(s+\|\Delta Q\|) \geq 0 .
\end{aligned}
$$

The assumptions in Theorem 8 are

$$
\begin{gathered}
\text { ass }_{4}=1-\sigma>0 \\
\text { ass }_{5}=\frac{(1-\sigma)^{2}}{\zeta+\sigma \zeta+2 \theta+2 \sqrt{((\zeta+\theta)(\sigma \zeta+\theta))}}-\epsilon>0 .
\end{gathered}
$$

By computation, we list them in Table 1.

The results listed in Table 1 show that the assumptions of Theorems 5 and 8 are satisfied.

By Theorems 5 and 8 , we can compute the relative perturbation bounds $\xi_{1}$ and $\xi_{2}=\nu /\|X\|$, respectively. These results averaged as the geometric mean of 10 randomly perturbed runs. Some results are listed in Table 2.

The results listed in Table 2 show that the perturbation bound $\xi_{2}$ given by Theorem 8 is fairly sharp, while the bound $\xi_{1}$ given by Theorem 5 which does not depend on the exact solution is conservative.

Example 16. We consider the matrix equation

$$
X-A_{1}^{*} X^{0.5} A_{1}-A_{2}^{*} X^{0.25} A_{2}=I,
$$

with

$$
\begin{aligned}
A_{1} & =\frac{(1 / 3)+2 \times 10^{-2}}{\|A\|} A, \\
A_{2} & =\frac{(1 / 6)+3 \times 10^{-2}}{\|A\|} A, \\
A & =\left(\begin{array}{lllll}
2 & 1 & 0 & 0 & 0 \\
1 & 2 & 1 & 0 & 0 \\
0 & 1 & 2 & 1 & 0 \\
0 & 0 & 1 & 2 & 1 \\
0 & 0 & 0 & 1 & 2
\end{array}\right) .
\end{aligned}
$$

Choose $\widetilde{X}_{1}=A, \widetilde{X}_{2}=2 A$. Let the approximate solution $\widetilde{X}_{k}$ of $X$ be given with the iterative method (6), where $k$ is the iteration number.

The residual $R\left(\widetilde{X}_{k}\right) \equiv I+A_{1}^{*} \widetilde{X}_{k}^{0.5} A_{1}+A_{2}^{*} \widetilde{X}_{k}^{0.25} A_{2}-\widetilde{X}_{k}$ satisfies the conditions in Theorem 10.

By Theorem 10, we can compute the backward error bound for $\widetilde{X}_{k}$ as follows:

$$
\left\|\widetilde{X}_{k}-X\right\| \leq \mu\left\|R\left(\widetilde{X}_{k}\right)\right\|
$$

where

$$
\begin{gathered}
\mu=\frac{2\left\|\widetilde{X}_{k}\right\|\left\|\widetilde{X}_{k}^{-1}\right\|}{\theta_{1}+\sqrt{\theta_{1}^{2}-4\left\|\widetilde{X}_{k}^{-1}\right\|\left\|R\left(\widetilde{X}_{k}\right)\right\|}}, \\
\theta_{1} \equiv 1+\left\|\widetilde{X}_{k}^{-1}\right\|\left\|R\left(\widetilde{X}_{k}\right)\right\| \\
-\left(0.5\left\|\widetilde{X}_{k}^{1 / 4} A_{1} \widetilde{X}_{k}^{-1 / 2}\right\|^{2}\right. \\
\left.+0.25\left\|\widetilde{X}_{k}^{1 / 8} A_{2} \widetilde{X}_{k}^{-1 / 2}\right\|^{2}\right) .
\end{gathered}
$$

Some results are listed in Table 3.

The results listed in Table 3 show that the error bound given by Theorem 10 is fairly sharp.

Example 17. We study the matrix equation

$$
X-A_{1}^{*} X^{1 / 2} A_{1}-A_{2}^{*} X^{1 / 3} A_{2}=Q,
$$

with

$$
\begin{aligned}
A_{1} & =\left(\begin{array}{cc}
0 & 0.55+10^{-k} \\
0 & 0
\end{array}\right), \\
A_{2} & =\frac{1}{2} A_{1}, \\
Q & =\left(\begin{array}{ll}
1 & 1 \\
0 & 1
\end{array}\right) .
\end{aligned}
$$

By Remark 14, we can compute the relative condition number $c_{\text {rel }}(X)$. Some results are listed in Table 4.

Table 4 shows that the unique positive definite solution $X$ is well conditioned. 
TABLE 2: Perturbation bounds for Example 15 with different values of $j$.

\begin{tabular}{lcccc}
\hline$j$ & 4 & 5 & 6 & 7 \\
$\|\widetilde{X}-X\| /\|X\|$ & $2.5627 \times 10^{-5}$ & $3.8447 \times 10^{-6}$ & $5.1681 \times 10^{-7}$ & $2.1776 \times 10^{-8}$ \\
$\xi_{1}$ & $2.1885 \times 10^{-4}$ & $1.9891 \times 10^{-5}$ & $2.4026 \times 10^{-6}$ & $1.8251 \times 10^{-7}$ \\
$\xi_{2}$ & $8.0828 \times 10^{-5}$ & $7.4741 \times 10^{-6}$ & $8.8011 \times 10^{-7}$ & $6.9496 \times 10^{-8}$ \\
\hline
\end{tabular}

TABLE 3: Backward error bound for Example 16 with different values of $k$.

\begin{tabular}{lcccc}
\hline$k$ & 8 & 10 & 12 & 14 \\
\hline$\left\|\widetilde{X}_{k}-X\right\|$ & $6.1091 \times 10^{-4}$ & $4.0865 \times 10^{-5}$ & $2.6837 \times 10^{-6}$ & $1.7372 \times 10^{-7}$ \\
$\mu \| R\left(\widetilde{X}_{k}\right)$ & $7.1435 \times 10^{-4}$ & $4.7784 \times 10^{-5}$ & $3.1381 \times 10^{-6}$ & $2.0318 \times 10^{-7}$ \\
\hline
\end{tabular}

TABLE 4: Relative condition number for Example 17 with different values of $k$.

\begin{tabular}{lccccc}
\hline$k$ & 1 & 3 & 5 & 7 & 9 \\
\hline$c_{\text {rel }}(X)$ & 1.0717 & 1.0228 & 1.0225 & 1.0225 & 1.0225 \\
\hline
\end{tabular}

\section{Acknowledgments}

The author wishes to express her gratitude to the referees for their fruitful comments. The work was supported in part by National Nature Science Foundation of China (11201263), Natural Science Foundation of Shandong Province (ZR2012AQ004), and Independent Innovation Foundation of Shandong University (IIFSDU), China.

\section{References}

[1] B. L. Buzbee, G. H. Golub, and C. W. Nielson, "On direct methods for solving Poisson's equations," SIAM Journal on Numerical Analysis, vol. 7, pp. 627-656, 1970.

[2] W. N. Anderson Jr., G. B. Kleindorfer, P. R. Kleindorfer, and M. B. Woodroofe, "Consistent estimates of the parameters of a linear system," Annals of Mathematical Statistics, vol. 40, pp. 2064-2075, 1969.

[3] W. N. Anderson, T. D. Morley, and G. E. Trapp, "The cascade limit, the shorted operator and quadratic optimalcontrol," in Linear Circuits, Systems and Signal Processsing: Theory and Application, C. I. Byrnes, F. C. Martin, and R. E. Saeks, Eds., pp. 3-7, North-Holland, New York, NY, USA, 1988.

[4] R. S. Bucy, "A priori bounds for the Riccati equation," in Proceedings of the 6th Berkeley Symposium on Mathematical Statistics and Probability, vol. 3 of Probability Theory, pp. 645656, University of California, Berkeley, Calif, USA, 1972.

[5] D. V. Ouellette, "Schur complements and statistics," Linear Algebra and its Applications, vol. 36, pp. 187-295, 1981.

[6] W. Pusz and S. L. Woronowicz, "Functional calculus for sesquilinear forms and the purification map," Reports on Mathematical Physics, vol. 8, no. 2, pp. 159-170, 1975.

[7] J. Zabczyk, "Remarks on the control of discrete-time distributed parameter systems," SIAM Journal on Control and Optimization, vol. 12, pp. 721-735, 1974.

[8] M. Berzig, X. F. Duan, and B. Samet, "Positive definite solution of the matrix equation $X=Q-A^{*} X^{-1} A+B^{*} X^{-1} B$ via BhaskarLakshmikantham fixed point theorem," Mathematical Sciences, vol. 6 , article $27,2012$.
[9] J. Cai and G. L. Chen, "On the Hermitian positive definite solution of nonlinear matrix equation $X^{s}+A^{*} X^{-t} A=Q$," Applied Mathematics and Computation, vol. 217, pp. 2448-2456, 2010.

[10] X. Duan and A. Liao, "On the existence of Hermitian positive definite solutions of the matrix equation $X^{s}+A^{*} X^{-t} A=Q$," Linear Algebra and its Applications, vol. 429, no. 4, pp. 673-687, 2008.

[11] X. Duan, C. Li, and A. Liao, "Solutions and perturbation analsis for the nonlinear matrix equation $X+\sum_{i=1}^{m} A_{i}^{*} X^{-1} A_{i}=I$," Applied Mathematics and Computation, vol. 218, no. 8, pp. 44584466, 2011.

[12] J. C. Engwerda, "On the existence of a positive definite solution of the matrix equation $X+A^{T} X^{-1} A=I$," Linear Algebra and its Applications, vol. 194, pp. 91-108, 1993.

[13] A. Ferrante and B. C. Levy, "Hermitian solutions of the equation $X=Q+N X^{-1} N^{*}$, Linear Algebra and its Applications, vol. 247, pp. 359-373, 1996.

[14] C.-H. Guo and P. Lancaster, "Iterative solution of two matrix equations," Mathematics of Computation, vol. 68, no. 228, pp. $1589-1603,1999$.

[15] V. I. Hasanov, "Positive definite solutions of the matrix equations $X \pm A^{*} X^{-q} A=Q$," Linear Algebra and its Applications, vol. 404, pp. 166-182, 2005.

[16] V. I. Hasanov and I. G. Ivanov, "On two perturbation estimates of the extreme solutions to the equations $X \pm A^{*} X^{-1} A=Q$," Linear Algebra and its Applications, vol. 413, no. 1, pp. 81-92, 2006.

[17] V. I. Hasanov, I. G. Ivanov, and F. Uhlig, "Improved perturbation estimates for the matrix equations $X \pm A^{*} X^{-1} A=Q$," Linear Algebra and its Applications, vol. 379, pp. 113-135, 2004, Tenth Conference of the International Linear Algebra Society.

[18] Y.-M. He and J.-H. Long, "On the Hermitian positive definite solution of the nonlinear matrix equation $X+\sum_{i=1}^{m} A_{i}^{*} X^{-1} A_{i}$," Applied Mathematics and Computation, vol. 216, no. 12, pp. 3480-3485, 2010.

[19] I. G. Ivanov and S. M. El-sayed, "Properties of positive definite solutions of the equation $X+A^{*} X^{-2} A=I$," Linear Algebra and its Applications, vol. 279, no. 1-3, pp. 303-316, 1998.

[20] I. G. Ivanov, V. I. Hasanov, and B. V. Minchev, "On matrix equations $X \pm A^{*} X^{-2} A=I$, Linear Algebra and its Applications, vol. 326, no. 1-3, pp. 27-44, 2001.

[21] J. Li and Y. H. Zhang, "The Hermitian positive definite solution and its perturbation analysis for the matrix equation $X-$ $A^{*} X^{-1} A=Q$," Mathematica Numerica Sinica, vol. 30, no. 2, pp. 129-142, 2008 (Chinese). 
[22] J. Li and Y. Zhang, "Perturbation analysis of the matrix equation $X-A^{*} X^{-q} A=Q$," Linear Algebra and its Applications, vol. 431, no. 9, pp. 1489-1501, 2009.

[23] X.-G. Liu and H. Gao, "On the positive definite solutions of the matrix equations $X^{s} \pm A^{T} X^{-t} A=I_{n}$," Linear Algebra and its Applications, vol. 368, pp. 83-97, 2003.

[24] J.-G. Sun and S.-F. Xu, "Perturbation analysis of maximal solution of the matrix equation $X+A^{*} X^{-1} A=P$. II," Linear Algebra and its Applications, vol. 362, pp. 211-228, 2003.

[25] H. Xiao and J. T. Wang, "On the matrix equation $X-A^{*} X^{-p} A=$ $Q(p>1)$," Chinese Journal of Engineering Mathematics, vol. 26, no. 2, pp. 305-309, 2009.

[26] S.-F. Xu, "Perturbation analysis of the maximal solution of the matrix equation $X+A^{*} X^{-1} A=P$, Linear Algebra and its Applications, vol. 336, pp. 61-70, 2001.

[27] X. Yin, S. Liu, and L. Fang, "Solutions and perturbation estimates for the matrix equation $X^{s}+A^{*} X^{-t} A=Q$," Linear Algebra and its Applications, vol. 431, no. 9, pp. 1409-1421, 2009.

[28] X. Yin, S. Liu, and T. Li, "On positive definite solutions of the matrix equation $X+A^{*} X^{-q} A=Q(0<q \leq 1)$," Taiwanese Journal of Mathematics, vol. 16, no. 4, pp. 1391-1407, 2012.

[29] X. Zhan, "Computing the extremal positive definite solutions of a matrix equation," SIAM Journal on Scientific Computing, vol. 17, no. 5, pp. 1167-1174, 1996.

[30] X. Zhan and J. Xie, "On the matrix equation $X+A^{T} X^{-1} A=I$," Linear Algebra and its Applications, vol. 247, pp. 337-345, 1996.

[31] Y. Zhang, "On Hermitian positive definite solutions of matrix equation $X+A^{*} X^{-2} A=I$, Linear Algebra and its Applications, vol. 372, pp. 295-304, 2003.

[32] Y.-H. Zhang, "On Hermitian positive definite solutions of matrix equation $X-A^{*} X^{-2} A=I$," Journal of Computational Mathematics, vol. 23, no. 4, pp. 408-418, 2005.

[33] G. Jia and D. Gao, "Perturbation estimates for the nonlinear matrix equation $X-A^{*} X^{q} A=Q(0<q<1)$," Journal of Applied Mathematics and Computing, vol. 35, no. 1-2, pp. 295304, 2011.

[34] X. Duan, A. Liao, and B. Tang, "On the nonlinear matrix equation $X-\sum_{i=1}^{m} A_{i}^{*} X^{\delta_{i}} A_{i}=Q$," Linear Algebra and its Applications, vol. 429, no. 1, pp. 110-121, 2008.

[35] X. Zhan, Matrix Inequalities, vol. 1790 of Lecture Notes in Mathematics, Springer, Berlin, Germany, 2002.

[36] J. R. Rice, "A theory of condition," SIAM Journal on Numerical Analysis, vol. 3, no. 2, pp. 287-310, 1966. 


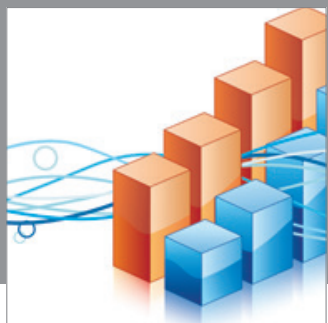

Advances in

Operations Research

mansans

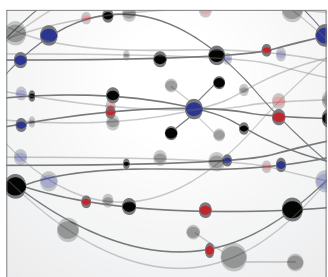

The Scientific World Journal
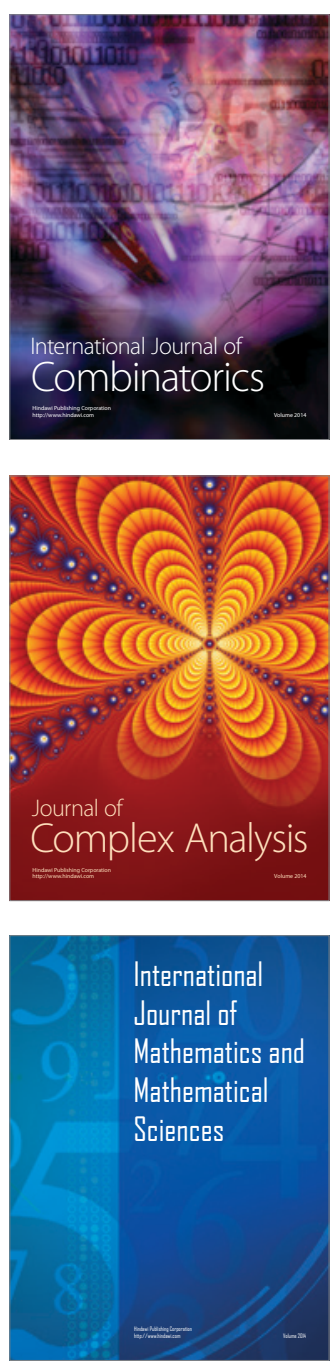
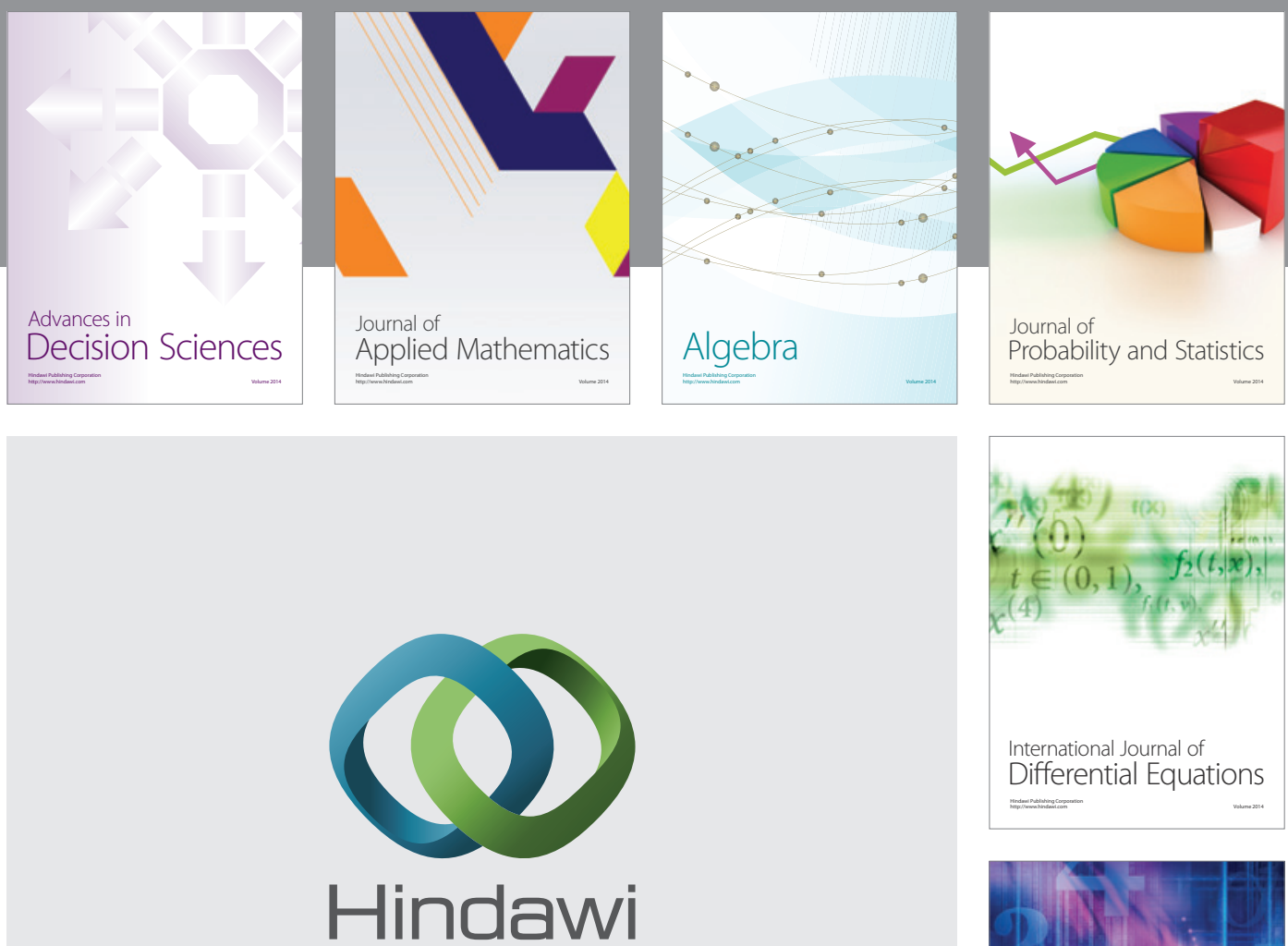

Submit your manuscripts at http://www.hindawi.com
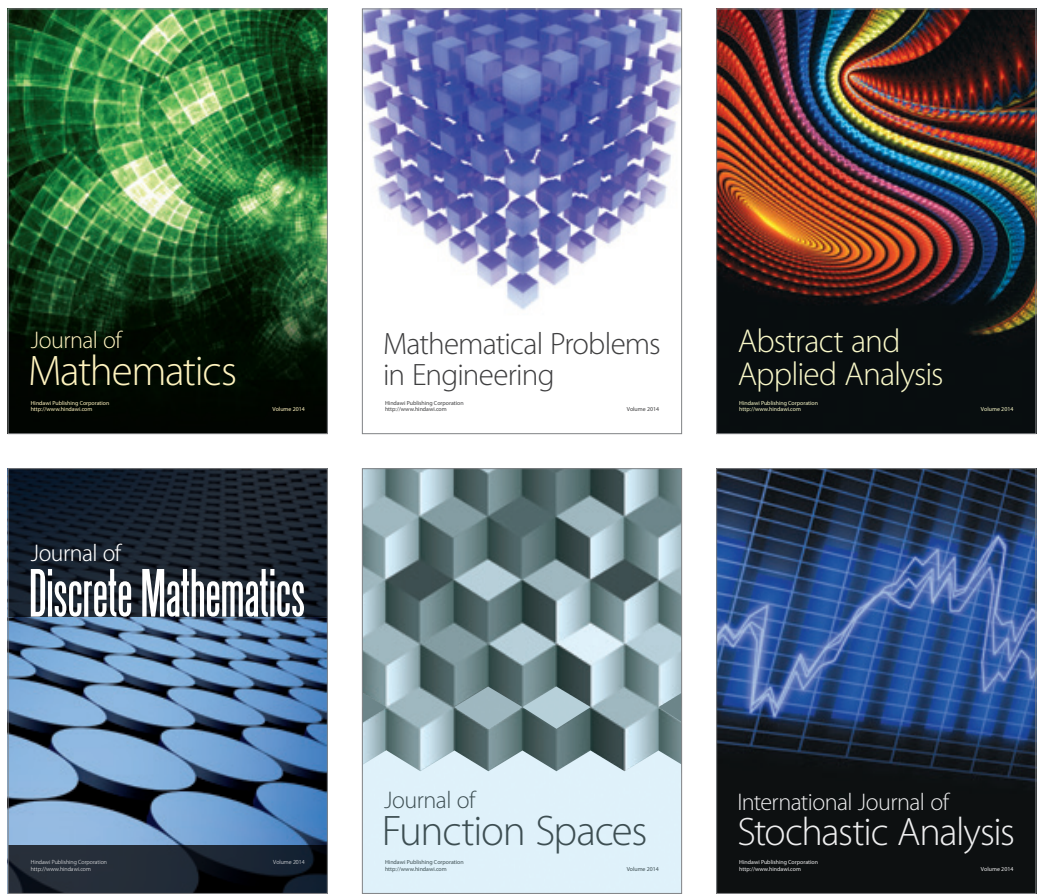

Journal of

Function Spaces

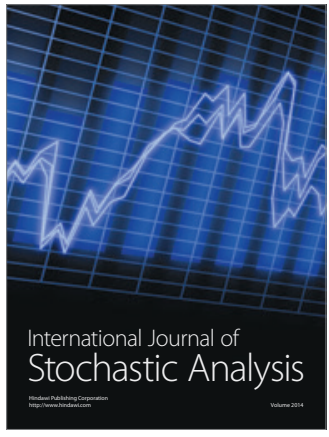

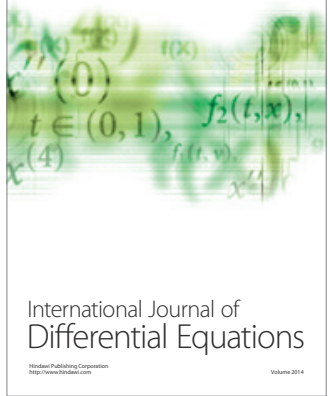
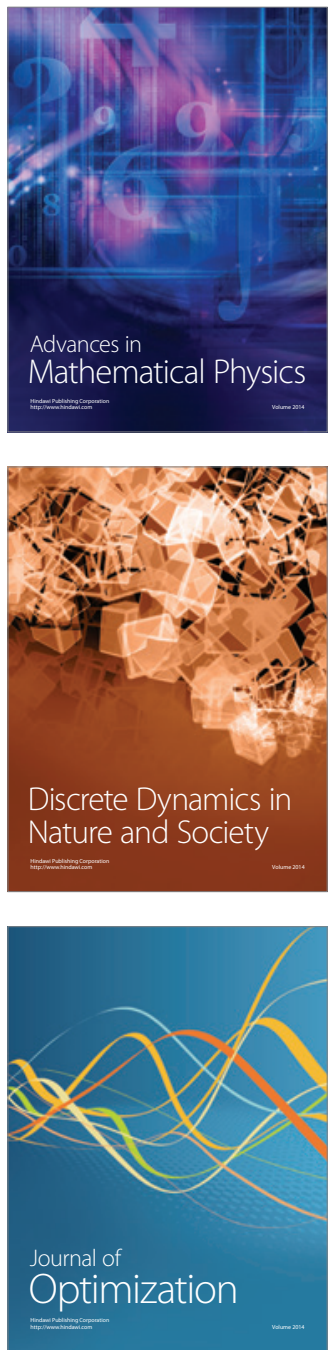